\title{
Effectiveness of Doppler Image of the Vertebral Artery as an Anatomical Landmark for Identification of Ultrasound-Guided Target Level in Cervical Spine
}

\author{
Dong-Hyuk Choi, Heun-Guyn Jung, Jeong-Ho Lee, Ji-Hoon Park, Yong-Soo Choi \\ Department of Orthopaedic Surgery, Kwangju Christian Hospital, Gwangju, Korea
}

\begin{abstract}
Study Design: A prospective sonographic study.
Purpose: To verify the effectiveness of simultaneous application of two landmarks, Doppler image of the vertebral artery and shape of the transverse tubercle of the seventh cervical (C7) vertebra.

Overview of Literature: Counting upwards from the C7 vertebra which only has a posterior tubercle of the transverse process is a commonly used method for ultrasound-guided cervical nerve root block. However, each transverse process has a different shape.

Methods: Sonograms of 20 volunteers were examined. At first, we identified the $\mathrm{C} 7$ transverse process based on the presence of the vertebral artery without the anterior tubercle. The $\mathrm{C} 5$ and $\mathrm{C} 6$ transverse processes were identified based on the presence of anterior tubercle without the vertebral artery. Subsequently, we placed needles on the C5, C6, and C7 transverse processes and the location and direction of needles were confirmed by fluoroscopy.

Results: In the 120 segments, $93.3 \%$ of needles were placed correctly as desired; $97.5 \%$ of needles were placed on the $5 \mathrm{C}$ transverse process; $97.5 \%$ of needles were placed on the $\mathrm{C} 6$ transverse process; and $85.0 \%$ of needles were placed on the $\mathrm{C} 7$ transverse process, respectively. Both sides showed the same accuracy of $93.3 \%$.

Conclusions: Simultaneous application of Doppler image of the vertebral artery and shape of the C7 transverse tubercle showed 93.3\% accuracy in identifying the target cervical level. Therefore, Doppler image of the vertebral artery can be considered to be a useful landmark for ultrasound-guided cervical nerve root block.
\end{abstract}

Keywords: Cervical spine; Nerve block; Ultrasonography

\section{Introduction}

Traditionally, cervical nerve root block was performed under fluoroscopy for accurate positioning of the injection needle. A contrast agent was used to prevent intravascular injection [1-3]. However, despite strict guidelines for performing the intervention, serious complications such as cerebral infarction or spinal cord injury may occur due to injection into the radicular artery. This is because soft tissue, nerves, and blood vessels cannot be visualized during fluoroscopy. Another disadvantage is the inability to perform the procedure on an outpatient

Received Jan 7, 2015; Revised Feb 13, 2015; Accepted Feb 14, 2015

Corresponding author: Heun-Guyn Jung

Department of Orthopaedic Surgery, Kwangju Christian Hospital,

37 Yangnim-ro, Nam-gu, Gwangju 61661, Korea

Tel: +82-62-650-5064, Fax: +82-62-650-5066, E-mail: stemcellchoi@gmail.com 
basis due to the possibility of radiation exposure [4-7]. In contrast, ultrasound-guided cervical nerve root block provides the advantage of preventing injection into blood vessels by real-time observation of soft tissue, nerves, and blood vessels. In addition, it is a relatively simple procedure with no exposure to radiation [8]. However, there is a limitation in identifying the target cervical segment with ultrasound.

To determine the target cervical segment during cervical nerve root block, a method that counts cephalad starting from the ultrasonographic marker of the transverse process with a posterior tubercle and without an anterior tubercle of the seventh cervical (C7) vertebra is needed [9]. However, this procedure may be technically demanding and limited in accuracy due to the difference in the shape of the transverse process of each cervical segment.

Vertebral artery is the first branch of the subclavian artery. It passes anterior to the transverse process of C7, enters through the transverse foramen of $\mathrm{C} 6$, progresses towards the head, and enters the foramen magnum through the posteromedial side of the atlas. We hypothesized that we can increase the accuracy by incorporating the anatomical characteristics of the vertebral artery in the currently used method for determining the target cervical segment. The aim of this study was to report the results of research on the use of marking that can increase the accuracy in identifying the target cervical segment by using the reference point of $\mathrm{C} 7$ after locating the vertebral artery through Doppler imaging while performing ultrasound-guided cervical nerve root block.

\section{Materials and Methods}

This prospective study was conducted on 20 volunteers (10 males and 10 females). The mean age of participants was 31.1 years, and there were 10 men and 10 women. The participants fully understood how the image would be rechecked with C-arm fluoroscopy after marking the target cervical segment using ultrasound, and agreed to participate in this study. It was performed on a total of 120 segments including C5, C6, and C7 on both sides of 20 participants.

\section{A method for counting the cervical segment by ultra- sound}

First, the patient was asked to lie in the supine position on a radiolucent table and pads were placed under both shoulders to extend the cervical spine by approximately 20 degrees. Subsequently, the neck was rotated externally by approximately 30 degrees contralateral to the targeted direction (Fig. 1). We positioned the high-resolution linear probe (12-MHz SONOs, LogiQ S7 Expert, GE healthcare, Wauwatosa, WI, USA) in the transverse direction at approximately $2 \mathrm{~cm}$ under the cricoid cartilage and identified the thyroid isthmus. The probe was moved to the lateral side in the horizontal direction to identify the transverse process of the cervical vertebrae after locating the thyroid isthmus. Thereafter, we moved the probe caudally, and defined the C7 segment based on Doppler image of the vertebral artery and anatomical characteristics of the transverse process with a posterior tubercle but no anterior tubercle. Next, a metal needle was attached to the skin to mark the $\mathrm{C} 7$ segment in the same axis of the linear probe, which is the same direction as that of the needle, while performing ultrasound-guided cervical selective nerve root block (Fig. 2A).

After moving the linear probe upward in the coronal plane, the upper cervical segment was defined as the C6 segment based on Doppler image of absence of the vertebral artery and anatomical findings of a transverse process which had both anterior and posterior tubercles (Fig. 2B). A metal needle was attached in the same manner as described previously after defining the transverse process of the C6 vertebra. Afterwards, the linear probe was moved upward in the coronal plane and we defined the C5 segment based on the findings which were same as those of the C6. A metal needle was attached to the skin in the same manner (Fig. 2C).

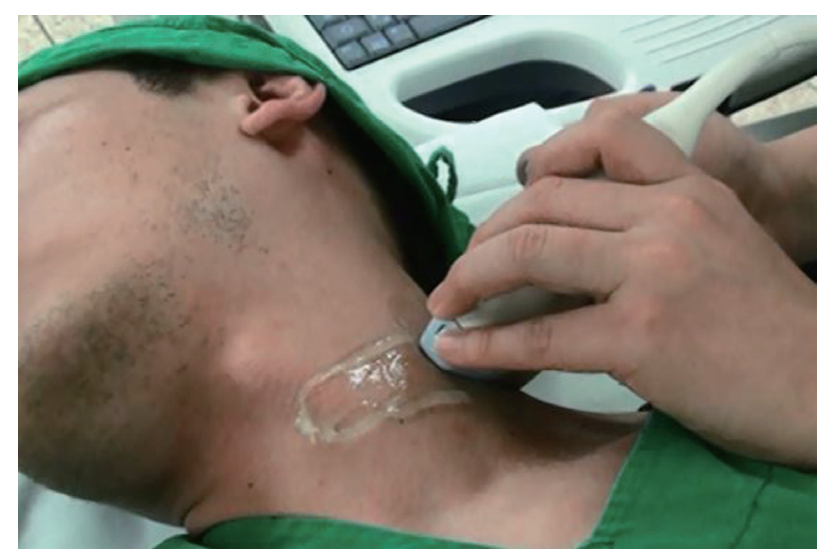

Fig. 1. Position for ultrasound-guided cervical selective nerve root block. Supine position with neck extension and rotation contralaterally. 


\section{Confirmation of the metal needle position using fluoroscopy}

An anteroposterior radiograph was obtained using $\mathrm{C}$-arm fluoroscopy without changing the position of the patient so as to maintain the same position as during ultrasound-guided cervical selective nerve root block. Location of the metal needle was confirmed on anteroposterior radiographs and the accuracy in identifying the target cervical segment was evaluated by an imaginary extension line of the metal needle (Fig. 3).
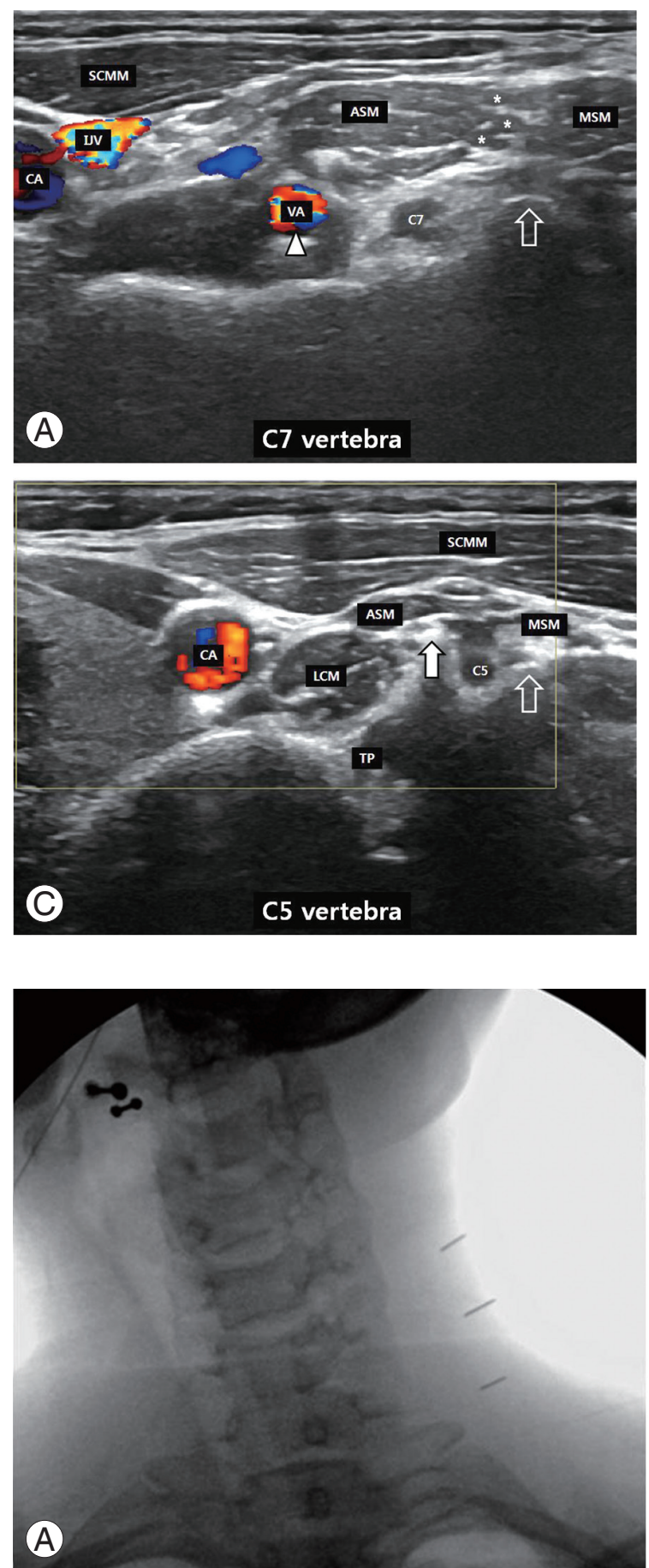

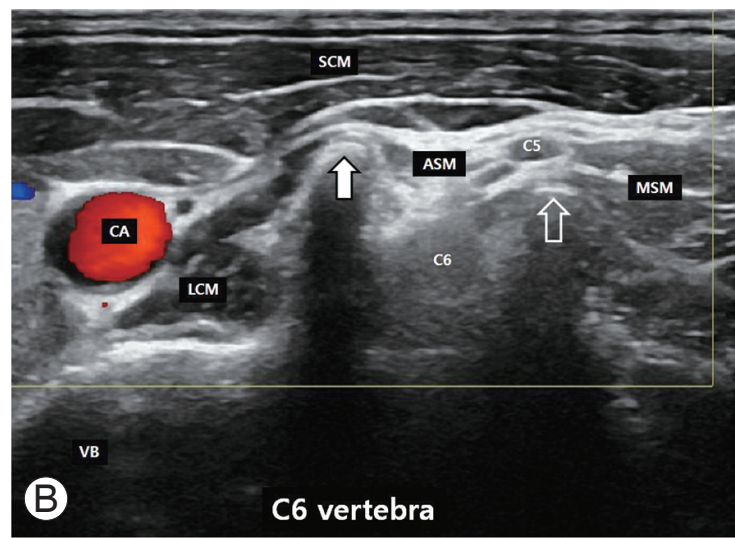

Fig. 2. Ultrasound image of the lower cervical spine. (A) C7 vertebra. Vertebral artery (arrowhead) is detected in front of the posterior tubercle (transparent arrow) of the C7 transverse process. (B, C) C6 and C5 vertebrae. Anterior (white arrow) and posterior (transparent arrow) tubercles are present, but the vertebral artery is not detected. C5, C6, and C7 indicate each nerve root. SCMM, sternocleidomastoid muscle; IJV, internal jugular vein; CA, carotid artery; ASM, anterior scalene muscle; VA, vertebral artery; MSM, middle scalene muscle; LCM, longus colli muscle; VB, vertebral body; TP, transverse process.

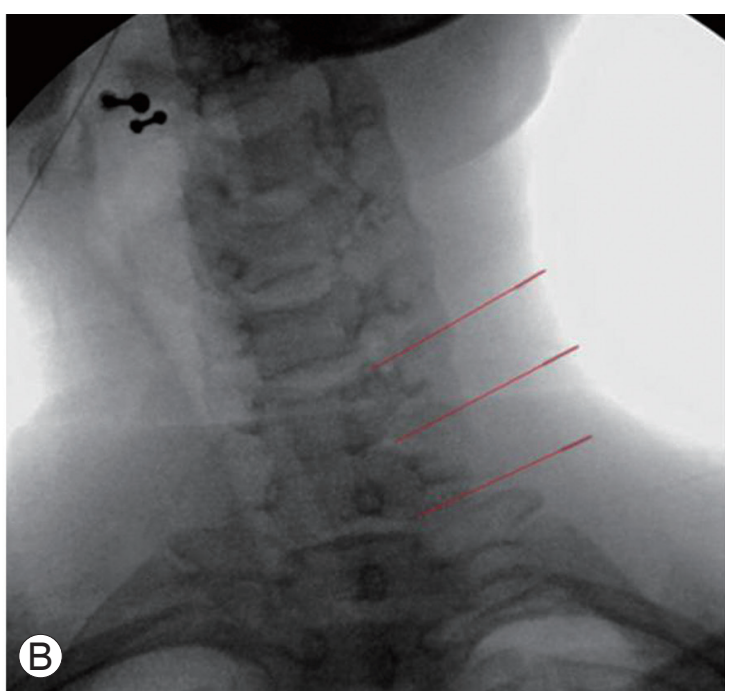

Fig. 3. Confirmation by C-arm fluoroscopy. (A) Needles were located at the target cervical segment. (B) Needles accurately indicated the target intervertebral foramen. 
Table 1. Validation of ultrasound-guided cervical target level confirmed by fluoroscopy

\begin{tabular}{lllc} 
Segment & Right & Left & Total \\
C5 & $20(100)$ & $19(95)$ & $39(97.5)$ \\
C6 & $19(95)$ & $20(100)$ & $39(97.5)$ \\
C7 & $17(85)$ & $17(85)$ & $34(85)$ \\
Total & $56(93.3)$ & $56(93.3)$ & - \\
\hline
\end{tabular}

Values are presented as number (\%).

\section{Results}

Accurate location of the metal needles in the target cervical segment was observed in 112 out of the total 120 cases (93.3\%) through fluoroscopy; 39 out of the 40 cases (97.5\%) in the C5 segment, 39 out of the 40 cases (97.5\%) in the C6 segment, and 34 out of the 40 cases (85\%) in the C7 segment, respectively (Table 1 ). There was no difference between both sides; 56 out of the 60 cases (93.3\%) in the left side and 56 out of the 60 cases (93.3\%) in the right side (Table 1). In the case of the vertebral artery which can be identified anterior to the $\mathrm{C} 7$ transverse process, 17 out of the 20 cases (85\%) in the left side and 17 out of the 20 cases $(85 \%)$ in the right side were identified.

The metal needle was located accurately in the target cervical segment based on the presence of the vertebral artery in two out of the five participants in whom the C7 transverse process could not be identified due to short neck length. The metal needle in the remaining three participants was located near the target cervical segment, but the imaginary extension line did not accurately indicate the target cervical segment (Fig. 4).

\section{Discussion}

Nowadays, ultrasound-guided cervical nerve root block is widely used because of its advantages; it can avoid intravascular injection or nerve damage due to real-time observation. There is no radiation exposure, and it is a relatively simple procedure that can be performed on an outpatient basis.

Two methods have been used to count each cervical segment during ultrasound-guided cervical intervention. First, the target cervical segment can be identified based on the transverse processes of lower cervical vertebrae. Anatomical characteristics were used to identify the target cervical segment with anterior and posterior tubercles of the transverse process of C6 but only a posterior tubercle of C7 [9]. Martinoli et al. [10] reported the difficulties in confirming anterior and posterior tubercles through
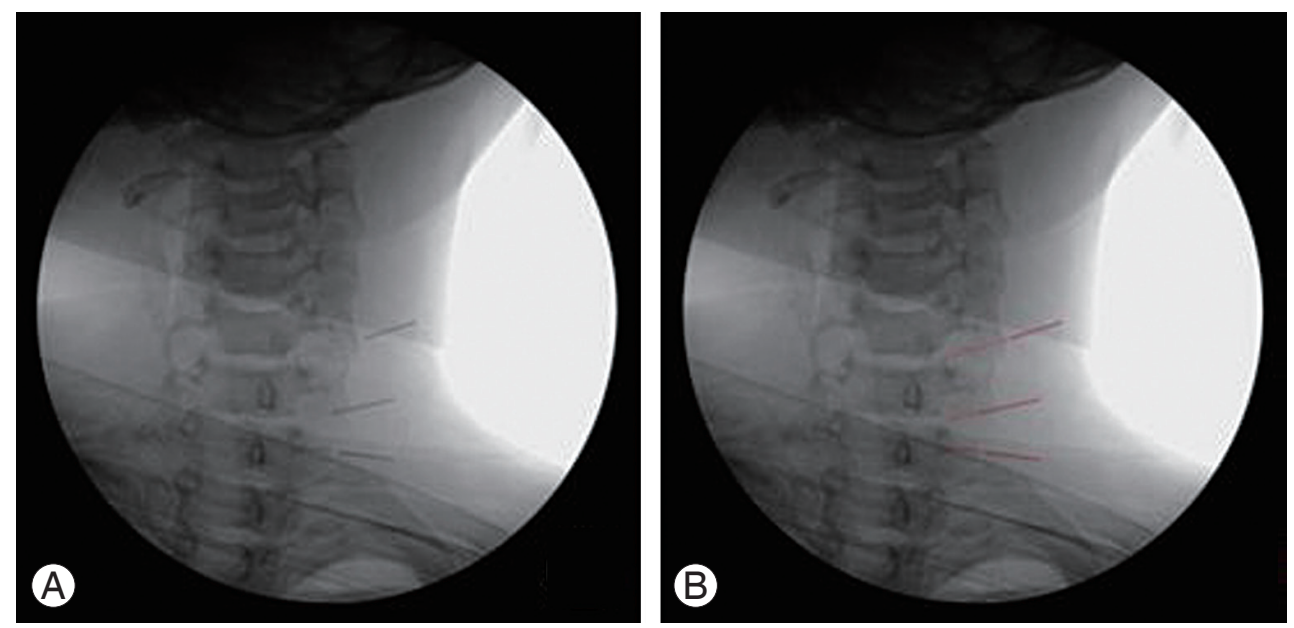

Fig. 4. Confirmation by C-arm fluoroscopy. (A) Needles were located near target cervical segment. (B) But, lowest needle does not indicate C7-T1 intervertebral foramen accurately. 
ultrasound since the transverse processes of lower cervical vertebrae are located deep in the soft tissue in patients with short and thick necks while performing ultrasoundguided cervical nerve root block in 20 patients.

The second method of counting the cervical segment is to perform a scan in the longitudinal axis parallel to the spine and place the mastoid on the side of the head, and to count the cervical segment by going down caudally. The probe is moved horizontally after determining the presence of the vertebral artery between $\mathrm{C} 1$ and $\mathrm{C} 2$, and the posterior facets of $\mathrm{C} 2$ and $\mathrm{C} 3$ can be identified on the ultrasound image. At this time, the target cervical segment can be identified by moving the probe downwards from $\mathrm{C} 2$, which shows the ultrasonographic finding of loss of hyperechoic pillar at the cephalic side [11]. However, it is difficult to ascertain ultrasound findings in cases with accompanying degenerative changes or presence of osteophytes, accompanying posterior angulation of the pillar, and accompanying narrow pillar due to short length of C2. Finlayson et al. [11] reported that the injection needle was located in the lateral margin of the targeted pillar in $19.1 \%$ of the cases while performing ultrasound-guided cervical nerve root block.

The two methods mentioned above have a limitation in identifying the target cervical segment, and therefore, we felt that there was a need for an additional marker to increase the accuracy in determining the target cervical segment by ultrasound. In this study, accuracy in identifying the target lower cervical segment could be increased by using anatomical characteristics of the vertebral artery that passes anterior to the $\mathrm{C} 7$ transverse process and runs through the C6 transverse foramen.

In previous studies, Narouze et al. [12] reported that all injection needles were located within $5 \mathrm{~mm}$ of target points in a prospective study of ten patients. Also, injury to the blood vessels and nerves could be avoided because progression of the metal needle could be observed in real time. On the other hand, Finlayson et al. [11] reported that the needles were located in the adjacent cervical segment, not in the target cervical segment in two patients, in spite of a high success rate of $94.5 \%$ in their study of 163 cases in 50 patients.

In this study, the target cervical segment was identified by the first method of using an additional ultrasonographic marker of the vertebral artery through a Doppler image. The metal needle was located accurately in the target cervical segment in 39 out of the 40 cases (97.5\%) in the $\mathrm{C} 5$ and $\mathrm{C} 6$ segments each, but the metal needle was located accurately in the target cervical segment in only 34 out of the 40 cases (85\%) in the C7 segment. This was because the soft tissue and muscle were thicker towards the lower cervical segment, especially in subjects with a short neck, and it was considered that a clear image could not be obtained through ultrasound. In addition, the metal needle was located accurately based on confirmation of the presence of the vertebral artery in two out of the five subjects, in whom it was difficult to identify the posterior tubercle of C7 through ultrasound, and the metal needle was located accurately in these two subjects; therefore, the accuracy rate for identifying the C7 segment was increased by $10 \%$ ( 2 out of the 20 cases). However, the vertebral artery was identified in 17 out of the 20 cases (85\%) in the left and right sides of the C7 segment each. In a previous study, Bruneau et al. [13] reported that in 93\% of the cases, the vertebral artery entered through the transverse foramen of $\mathrm{C} 6$, and in $1.0 \%, 5.0 \%$, and $0.8 \%$ of the cases, the vertebral artery entered through the transverse foramen of C4, C5, and C6, respectively. Therefore, for identifying the target cervical segment based on the presence of the vertebral artery as an ultrasonographic marker of $\mathrm{C} 7$, it is necessary to combine the anatomical characteristics of the transverse process of the $\mathrm{C} 7$ vertebra and Doppler image of the vertebral artery.

Ultrasound-guided cervical nerve root block is technically demanding, and therefore, a learning curve is necessary. When ultrasound-guided cervical nerve root block is performed by an unskilled physician, it is necessary to follow a practice of obtaining confirmation with fluoroscopy after ultrasound-guided contrast injection at the target cervical nerve root. After all, minimizing the complications associated with injections could be achieved with practice.

This study was not conducted in patients who had actual cervical disease and it only evaluated the accuracy in identifying the target cervical segment. Hence, there was a limitation of not evaluating the effect or complications which can occur after performing cervical nerve root block. Therefore, a further study should be performed for evaluating the efficacy of nerve root block in patients with actual cervical disease.

\section{Conclusions}

During ultrasound-guided cervical selective nerve root 
block, only 30 out of the 40 cases (75\%) were accurately located in the target cervical segment based on the anatomical finding of C7 transverse process with only a posterior tubercle but no anterior tubercle, and the reason for this low percentage of cases is the difficulty in identifying the transverse process of the C7 segment in patients with a short neck. However, the accuracy increased by 10\% (4 out of the 40 cases) through the Doppler image of the vertebral artery located in the C7 segment. The accuracy in identifying the target segment was $93.3 \%$ (112 out of the 120 cases) by simultaneous application of the anatomical finding of the C7 transverse process and Doppler image of the vertebral artery. This suggests that Doppler image of the vertebral artery can be a useful additional marker to identify the target cervical segment.

\section{Conflict of Interest}

No potential conflict of interest relevant to this article was reported.

\section{References}

1. Rathmell JP, Aprill C, Bogduk N. Cervical transforaminal injection of steroids. Anesthesiology 2004; 100:1595-600.

2. Vallee JN, Feydy A, Carlier RY, et al. Chronic cervical radiculopathy: lateral-approach periradicular corticosteroid injection. Radiology 2001;218:886-92.

3. Cyteval C, Thomas E, Decoux E, et al. Cervical radiculopathy: open study on percutaneous periradicular foraminal steroid infiltration performed under CT control in 30 patients. AJNR Am J Neuroradiol 2004; 25:441-5.

4. Tiso RL, Cutler T, Catania JA, Whalen K. Adverse central nervous system sequelae after selective transforaminal block: the role of corticosteroids. Spine J 2004;4:468-74.

5. Baker R, Dreyfuss P, Mercer S, Bogduk N. Cervical transforaminal injection of corticosteroids into a radicular artery: a possible mechanism for spinal cord injury. Pain 2003;103:211-5.

6. Provenzano DA, Fanciullo G. Cervical transforaminal epidural steroid injections: should we be performing them? Reg Anesth Pain Med 2007;32:168.

7. Scanlon GC, Moeller-Bertram T, Romanowsky SM, Wallace MS. Cervical transforaminal epidural steroid injections: more dangerous than we think? Spine (Phila Pa 1976) 2007;32:1249-56.

8. Fishman SM, Smith H, Meleger A, Seibert JA. Radiation safety in pain medicine. Reg Anesth Pain Med 2002;27:296-305.

9. Galiano K, Obwegeser AA, Bodner G, et al. Ultrasound-guided periradicular injections in the middle to lower cervical spine: an imaging study of a new approach. Reg Anesth Pain Med 2005;30:391-6.

10. Martinoli C, Bianchi S, Santacroce E, et al. Brachial plexus sonography: a technique for assessing the root level. AJR Am J Roentgenol 2002;179:699-702.

11. Finlayson RJ, Gupta G, Alhujairi M, et al. Cervical medial branch block: a novel technique using ultrasound guidance. Reg Anesth Pain Med 2012;37:21923.

12. Narouze SN, Vydyanathan A, Kapural L, et al. Ultrasound-guided cervical selective nerve root block: a fluoroscopy-controlled feasibility study. Reg Anesth Pain Med 2009;34:343-8.

13. Bruneau M, Cornelius JF, Marneffe V, et al. Anatomical variations of the V2 segment of the vertebral artery. Neurosurgery 2006;59(1 Suppl 1):ONS20-4. 